\title{
Calculating multivariate ruin probabilities via Gaver-Stehfest inversion technique
}

\author{
Miguel Usábel \\ Universidad Carlos III de Madrid, Dep. de Economía de la Empresa, Madrid, Spain and Dept. of Actuarial Mathematics and Statistics, \\ Heriot-Watt University, Edinburgh, UK
}

\begin{abstract}
Multivariate characteristics of risk processes are of high interest to academic actuaries. In such models, the probability of ruin is obtained not only by considering initial reserves $u$ but also the severity of ruin $y$ and the surplus before ruin $x$. This ruin probability can be expressed using an integral equation that can be efficiently solved using the Gaver-Stehfest method of inverting Laplace transforms. This approach can be considered to be an alternative to recursive methods previously used in actuarial literature.
\end{abstract}

JEL classification: G22; IM13; IM20

Keywords: Multivariate ultimate ruin probability; Laplace transform; Integral equations; Numerical methods

\section{Introduction}

Let us consider the classical risk process in continuous time $\left\{Z_{t}\right\}_{t>0}$ with $U_{k}$ claim sizes and premium $c$ per time unit,

$$
Z_{t}=u+c t-\sum_{k=1}^{N_{t}} U_{k},
$$

where $u$ is the initial reserves and $N_{\mathrm{t}}$ the total number of claims up to time $t$ following an homogeneous Poisson process of parameter $\lambda>0$. Let $B$ denote the distribution function of claim sizes $U_{k}$ with mean $\mu^{-1}$ and $c=$ $\lambda \mu^{-1}(1+\theta)$, where $\theta$ is the premium loading factor.

Let us now define $\tau=\inf \left\{t>0: Z_{t}<0\right\}$ as the ruin time and $Y=-Z_{\tau}$ as the deficit at ruin time or severity of ruin and $(X+u)=Z_{\tau^{-}}$as the surplus just before the ruin $(X>0)$.

The probability of ultimate ruin with initial reserves $u$, parameter $\lambda$ and severity of ruin less than $y$ and surplus less than $x+u$ is defined,

$$
P\{\tau<\infty, \quad X \leq x, \quad Y \leq y\}=\Psi_{x, y}(u) .
$$


We will now use the results obtained by Dufresne and Gerber (1988) and a similar renewal argument as in Frey and Schmidt (1996) and Gerber et al. (1987) in order to express the former probability with the following defective renewal equation:

$$
\Psi_{x, y}(u)=\frac{\lambda}{c} F_{x, y}(u)+\frac{\lambda}{c} \int_{0}^{u} \Psi_{x, y}(u-w) \mathrm{d} G(w),
$$

where $G(w)=\int_{0}^{w}(1-B(z)) \mathrm{d} z, g(w)=1-B(w)$ and

$$
F_{x, y}(w)=\int_{w}^{w+x}(B(z+y)-B(z)) \mathrm{d} z=\int_{w}^{w+x}(g(z)-g(z+y)) \mathrm{d} z
$$

for $x \in(0, \infty)$ and $y \in(0, \infty)$.

The result used by Frey and Schmidt (1996), expression (10), is just a particular case of the former approach when the premium loading factor is defined as

$$
\theta=\frac{1-\lambda \mu^{-1}}{\lambda \mu^{-1}}, \quad \theta>0
$$

Since the early 1980s, many methods have been developed in order to approximate $\Psi_{x, y}(u)$ (specially some particular interesting cases such as $x=\infty$ and/or $y=\infty$ ). They were based on a discretization of some aspect of the risk process and derived recursive expressions; see for example Dickson (1989), Dickson et al. (1995), Goovaerts and De Vylder (1984), Panjer (1986), Panjer and Wang (1993), Ramsay (1992b) and Ramsay and Usábel (1997). Panjer and Wang (1993) describe the conditions under which these recursions are stable.

Though some of these recursive approaches may be able to determine $\Psi_{x, y}(u)$ to any desired degree of accuracy, they are not suitable for heavy-tailed distributions, such as the Pareto or lognormal distribution for two reasons, citing Ramsay and Usábel (1997):

- To achieve a reasonable degree of accuracy, the interval of discretization must be at most one unit of the mean in length. If we standardize the unit of currency such that $\mu^{-1}=1$, then to obtain $\Psi_{x, y}(10)$ we must recursively estimate every intermediate unit point $\Psi_{x, y}(u)$ for $k=0,1,2, \ldots, 9,10$. This may be acceptable if we need only small values of $u$; however, for large values of $u$, say $u=500$ units, this method can be slow. For the Pareto, $\Psi_{x, y}(500)$ is not insignificant.

- The quadrature rules inherent in the recursive schemes are usually of low order. This further reduces its accuracy and its rate of convergence. To improve accuracy, the intervals of discretization are made even smaller. This substantially increases the number of intermediate calculations required, making the process of finding $\Psi_{x, y}(u)$ slower.

Before the shift to recursive methods explained in the last paragraph, the problem of ruin in the Collective Risk Theory had been extensively treated in actuarial literature using integral transforms in the restricted case when $y=\infty, x=\infty$; therefore not contemplating the multivariate case.

Since the paper by Sparre Andersen in 1955 many authors developed approximations for the ruin probability using Laplace-Stieltjes transforms. Cramér (1995) used the Wiener-Höpf method for the classical case and Thorin $(1970,1971,1973,1977)$ introduced the generalization when epochs of claims form a renewal process. Thorin and Wikstad (1973), Wikstad (1971, 1977) used Piessens (1969) inversion method of the Laplace transforms and Bohman (1971, 1974, 1975), focussed on inversions of Fourier transforms and Seal (1971, 1974) dealt with both Laplace and Fourier numerical inversions. Seal (1977) obtained an interesting result for the classical case and exponential claim size distribution using the Bromwich-Mellin inversion formula for Laplace transforms. As cited, numerical illustrations obtained using this methodology were based on Laplace transforms inversion techniques due to Piessens (1969) and Piessens and Branders (1971), in which complex analysis is involved. For other interesting work in actuarial literature using inversion of Laplace transforms see Covens et al. (1979). 
To our knowledge, this methodology has not been tested either in the context of approximating multivariate ruin probabilities or using Laplace transform inversion techniques involving only the evaluation of real numbers.

The objective of this paper is to present an alternative method of evaluating $\Psi_{x, y}(u)$, multivariate ruin probability in the classical case, based on the Gaver-Stehfest (GS) method of inverting Laplace transforms. We will show that this method used with classical standard computer languages (Pascal, FORTRAN, $C^{++}$) or mathematical assistants (MapleV, Mathematica or MathLab) can lead to very efficient approximations for the multivariate ultimate ruin probability.

The defective Volterra integral equation of the second kind ((1.1)) will then be solved numerically using the Laplace transform approach. In Section 2, Laplace transform of the multivariate ruin probability, $L\left(s, \Psi_{x, y}(u)\right.$ ), is studied and expressed as a function of the Laplace transform of the claim size distribution. In Section 3, we will introduce Gaver-Stehfest method of inverting Laplace transforms. This method is based on generating samples using generalized delta functions and accelerating the convergence using an extrapolation technique. Some interesting features concerning this method are discussed in Section 4. In Section 5, we deal with the actual use of GS method. Finally, in Section 6 we will include numerical illustrations showing that this method may be considered a very good alternative to recursive methods.

\section{Laplace transforms approach}

We will introduce now the so-called Laplace transform operator of a general function $\xi(w)$ with support in the non-negative real axis,

$$
L(s, \xi(w))=\int_{0}^{\infty} \mathrm{e}^{-s w} \xi(w) \mathrm{d} w, \quad \operatorname{Re}(s)>0
$$

applying it to (Eq (1.1)) and using the properties of Laplace transform we can get

$$
L\left(s, \Psi_{x, y}(u)\right)=\frac{\lambda}{c} L\left(s, F_{x, y}(u)\right)+\frac{\lambda}{c} L\left(s, \Psi_{x, y}(u)\right) L(s, g(x))
$$

and finally,

$$
L\left(s, \Psi_{x, y}(u)\right)=\frac{\left(\frac{\lambda}{c}\right) L\left(s, F_{x, y}(u)\right)}{1-\left(\frac{\lambda}{c}\right) L(s, g(u))}, \quad \operatorname{Re}(s)>0 .
$$

Using a basic property of Laplace transforms,

$$
L\left(s, F_{x, y}(u)\right)=\int_{0}^{\infty} \mathrm{e}^{-s u} \int_{u}^{u+x}(g(z)-g(y+z)) \mathrm{d} z \mathrm{~d} w=\frac{L\left(s, F_{x, y}^{\prime}(u)\right)+F_{x, y}(0)}{s},
$$

where

$$
F_{x, y}(0)=\int_{0}^{x}(g(z)-g(y+z)) \mathrm{d} z, \quad x>0 .
$$

Leibniz's differentiation theorem leads to

$$
\left.F_{x, y}^{\prime}(u)\right)=g(u+x)-g(u+x+y)+g(u+y)-g(u)
$$

and

$$
L\left(s, F_{x, y}^{\prime}(u)\right)=L(s, g(u+x))-L(s, g(u+x+y))+L(s, g(u+y))-L(s, g(u)),
$$


where

$$
\begin{aligned}
L(s, g(u+a)) & =\int_{0}^{\infty} \mathrm{e}^{-s u} g(u+a) \mathrm{d} u=\int_{0}^{\infty} \mathrm{e}^{-s u}(1-B(u+a)) \mathrm{d} u=\frac{1}{s}-L(s, B(u+a)) \\
& =\frac{1-B(a)-L(s, b(u+a))}{s} .
\end{aligned}
$$

After some arrangements we can get the following expressions and relations:

$$
\begin{aligned}
L\left(s, \Psi_{x, y}(u)\right)= & \left(\frac{1}{s}\right)\left(\frac{\mu^{-1}-L(s, g(u))}{\mu^{-1}(1+\theta)-L(s, g(u))}\right)-\left(\frac{1}{s}\right)\left(\frac{\int_{0}^{\infty} g(y+z) \mathrm{d} z-L(s, g(u+y))}{\mu^{-1}(1+\theta)-L(s, g(u))}\right) \\
& -\left(\frac{1}{s}\right)\left(\frac{\int_{x}^{\infty} g(z) \mathrm{d} z-L(s, g(u+x))}{\mu^{-1}(1+\theta)-L(s, g(u))}\right)+\left(\frac{1}{s}\right)\left(\frac{\int_{x}^{\infty} g(y+z) \mathrm{d} z-L(s, g(u+x+y))}{\mu^{-1}(1+\theta)-L(s, g(u))}\right) \\
L\left(s, \Psi_{\infty, \infty}(u)\right)= & \left(\frac{1}{s}\right)\left(\frac{\mu^{-1}-L(s, g(u))}{\mu^{-1}(1+\theta)-L(s, g(u))}\right) \\
L\left(s, \Psi_{\infty, y}(u)\right)= & L\left(s, \Psi_{\infty, \infty}(u)\right)-\left(\frac{1}{s}\right)\left(\frac{\int_{0}^{\infty} g(y+z) \mathrm{d} z-L(s, g(u+y))}{\mu^{-1}(1+\theta)-L(s, g(u))}\right) \\
L\left(s, \Psi_{x, \infty}(u)\right)= & L\left(s, \Psi_{\infty, \infty}(u)\right)-\left(\frac{1}{s}\right)\left(\frac{\int_{x}^{\infty} g(z) \mathrm{d} z-L(s, g(u+x))}{\mu^{-1}(1+\theta)-L(s, g(u))}\right) \\
L\left(s, \Psi_{x, y}(u)\right)= & L\left(s, \Psi_{\infty, y}(u)\right)+L\left(s, \Psi_{x, \infty}(u)\right)-L\left(s, \Psi_{\infty, \infty}(u)\right) \\
& +\left(\frac{1}{s}\right)\left(\frac{\int_{x}^{\infty} g(y+z) \mathrm{d} z-L(s, g(u+x+y))}{\mu^{-1}(1+\theta)-L(s, g(u))}\right)
\end{aligned}
$$

\section{Gaver-Stehfest method}

Once the expression of the Laplace transform is obtained, we will use the Gaver-Stehfest (GS) method to approximate the value of the multivariate ruin probability function.

Following Davies and Martin (1979), Gaver-Stehfest method can be classified in the context of numerical inversion procedures as a method which computes a sample,

$$
I_{n}(t)=\int_{0}^{\infty} \delta_{n}(t, u) \xi(u) \mathrm{d} u,
$$

where the functions $\delta_{n}(t, u)$ form a delta convergent sequence in the sense of Gelfand and Shilov (1964) which means that they have the property that $I_{n}(t)$ tends to $\xi(t)$ with increasing $n$. Gaver (1966) used the following family of generalized delta functions:

$$
\delta_{n}(t, u)=\left(\frac{\ln (2)}{t}\right)\left(\frac{(2 n) !}{n !(n-1) !}\right)\left(1-\mathrm{e}^{-\ln (2) t u}\right)^{n} \mathrm{e}^{-n \ln (2) t u}
$$

and the family of convergent approximations can be expressed,

$$
I_{n}(t)=\left(\frac{\ln (2)}{t}\right)\left(\frac{(2 n) !}{n !(n-1) !}\right) \sum_{i=0}^{n}{ }_{i}^{n}(-1)^{i} L\left(\frac{(n+i) \ln (2)}{t}, \xi(t)\right) .
$$


Gaver also proved the asymptotic expansion

$$
I_{n}(t) \sim \xi(t)+\frac{\alpha_{1}}{n}+\frac{\alpha_{2}}{n^{2}}+\cdots
$$

and used extrapolation to the limit to speed up the convergence.

Nevertheless, the most useful extrapolation formula has been derived by Stehfest (1970) leading to the formula

$$
\Psi_{x, y}(u)=\Psi_{x, y}^{N}(u)+(-1)^{N / 2+1} \frac{\alpha}{\left(\frac{N}{2}\right) !}+\mathrm{o}\left(\frac{1}{\left(\frac{N}{2}\right) !}\right)
$$

and the approximation of order $N$ is

$$
\Psi_{x, y}(u) \simeq \Psi_{x, y}^{N}(u)=z \sum_{n=1}^{N} k_{n}^{N} L\left(n z, \Psi_{x, y}(u)\right),
$$

where

$$
z=\frac{\ln (2)}{u}
$$

and

$$
k_{n}^{N}=(-1)^{n+N 2} \sum_{i=[(n+1) / 2]}^{\operatorname{Min}(n, N / 2)} \frac{i^{(N / 2)}(2 i) !}{\left(\frac{N}{2}-i\right) ! i !(i-1) !(n-i) !(2 i-n) !} .
$$

Stehfest (1970) also proved that the magnitude of the error is of order $((N / 2) !)^{-1}$.

We should also mention, citing Davies and Martin (1979), that Gaver-Stehfest method is a particular example of a general class of methods proposed by Zakian (1969).

\section{Some interesting features about Gaver-Stehfest method}

To our knowledge, the only use of Gaver-Stehfest method in actuarial literature is due to Dalpatadu et al. (1996) in the context of approximating the distribution of the total loss of a portfolio. The conclusion reached by these authors is that inverting Laplace transforms via GS method is shown to be a better approach than the recursive methods based on Panjer's recursive algorithm, extensively used in actuarial literature.

Let us state these remarks about the Gaver-Stehfest method:

- The use of Gaver-Stehfest is guaranteed to lead to very good approximations when the unknown function $\Psi_{x, y}(u)$ is smooth in the sense that it has no discontinuities, salient points, sharp peaks or rapid oscillations, avoiding the study of complex analysis features as poles or abscissa of convergence or asymptotic behavior of the Laplace transform function, see Davies and Martin (1979). Multivariate ruin probability functions $\Psi_{x, y}(u)$ can be considered as smooth in the terms mentioned above.

- Computational execution times are highly reliable in the case of Gaver-Stehfest method for two reasons. First, compared with other methods of inversion and for the same $N$ (for a complete information see Davies and Martin (1979), Table VI) we can say that GS method is about 50 times faster than methods based on Laguerre polynomials, 206 times than methods based on Chebyshev polynomials or 628 times than methods based on Fourier series approach. Second, the last statement is emphasized by the fact that $N$ should not be very large in order to get a very acceptable good accuracy (as we will show later in the numerical illustrations). As a result, methods based on Chebyshev polynomials and Fourier transforms with accelerating convergence cannot be recommended due to their significant disadvantage in terms of computational efficiency. 
- Methods based on the use of Laguerre polynomials by Piessens and Branders (1971) and Weeks (1966), together with those that use Chebyshev polynomials and Fourier transforms, are the only ones that could be considered, in general terms, more accurate than GS method in the comparative study by (Davies and Martin, 1979). Nevertheless, we should be cautious when considering the conclusions made by these authors. They only considered approximations for small values of the argument of the original function (actually 15 was the largest figure considered).

When approximating $\Psi_{x, y}(u)$ using Laguerre (or other families of orthogonal polynomials based methods) with large values of $u$, we found unstable behaviors or very slow convergence. It is important to state that when approximating ruin probabilities using heavy-tailed distributions of the claim size the use of large values of $u$ is significant. Seal (1975) already claimed that Laguerre series cannot be recommended as a practical method of numerical inversion of Laplace transform.

\section{Practical use of the Gaver-Stehfest method}

From Eq. (3.2)

$$
\Psi_{x, y}(u) \simeq \Psi_{x, y}^{N}(u)=z \sum_{n=1}^{N} k_{n}^{N} L\left(n, z, \Psi_{x, y}(u)\right) ; \quad z=\frac{\ln (2)}{u}
$$

it is easy to deduce that the weights used in the GS method, $k_{n}^{N}$ obtained using (3.3), are independent of the initial reserves or ruin function considered and we just need to evaluate the Laplace transform of the function considered $N$ times.

The main disadvantage of GS method is the increasing magnitude of the weights of the approximations, $k_{n}^{N}, n=$ $1,2, \ldots, N$, as $N$ increases, as we show in Table 1 . However, we should also mention that this is not at a comparative handicap compared with the methods based on orthogonal polynomials because the same problem arises. An exception could be considered the method by Piessens and Branders (1971), but although the weights remain small in magnitude, the values of polynomials become also very large for increasing values of the initial reserves $u$.

Classical programming languages such as Pascal or Fortran can usually work with about 20 significant digits (see for example Turbo Pascal Programmer's guide). As a consequence, if we want to achieve a degree of accuracy of

\begin{tabular}{ll} 
Table 1 & \\
\hline$N$ & $\operatorname{Max}\left\{k_{n}^{N}\right\}_{n=1}^{N}$ \\
\hline 2 & 2 \\
4 & 26 \\
6 & 810 \\
8 & 18730 \\
10 & 375911.6 \\
12 & $0.7 \times 10^{7}$ \\
14 & $0.1 \times 10^{9}$ \\
16 & $0.3 \times 10^{10}$ \\
18 & $0.7 \times 10^{11}$ \\
20 & $0.1 \times 10^{13}$ \\
22 & $0.3 \times 10^{14}$ \\
24 & $0.7 \times 10^{15}$ \\
26 & $0.1 \times 10^{17}$ \\
28 & $0.3 \times 10^{18}$ \\
30 & $0.8 \times 10^{19}$ \\
\hline
\end{tabular}


about 8 or 9 significant digits, the maximum $N$ that we could consider when approximating ought to be around 18 or 20 so that we can perform the operations involved in expression (3.2) with enough significant digits that guarantee that rounding off errors will not make the algorithm unstable due to substraction of nearly equal numbers.

Nevertheless, most commercial FORTRAN 77 or 90 PC implementations offer as an extension the type Real*16 allowing operations with around 30 significant digits. Moreover, arbitrary precision subroutines are a common and extensively used item in computer libraries such as GNU MP, see Bailey $(1993$, 1995). One can easily obtain (and implement) these practical and fast subroutines with just a free downloading at http://www.swox.com/gmp. Similar features can be also found for $C^{++}$interfaces. In this context of arbitrary precision, the advantages of using GS compared to other numerical techniques are fully displayed, mainly due to the convergence rate of the method $((N / 2) !)^{-1}$.

For those actuaries who are not familiar with computer programming and not very much concerned with execution times, the method recommended in this work to approximate multivariate ultimate ruin probabilities can be very easily implemented using mathematical assistants such as Maple V or Mathematica or MathLab. These programs, known and used by any actuarial student, allow also either arbitrary or very high (over 100 s.d.) precision.

\section{Numerical examples}

This approach was tested for Pareto and Log-Normal claim sizes distributions. Expressions (2.6)-(2.9) were used in the intermediate steps. Evaluation of integrals involving Pareto distribution were made using the exponential integral (see for example Gradshteyn and Ryzhik (1994), formula 3.353.2). Log-normal integrals expressions were approximated numerically using Clenshaw-Curtis quadrature routine.

Initially only 20 significant digits were considered in the calculations and, in order not to make the algorithm unstable, as mentioned in the former section, $N=18$. Results are displayed in Tables 2 and 4 . These tables include approximations of the multivariate ruin probability $\Psi_{x, y}(u)$ rounded to the 5th decimal place. Beside these figures, in brackets, the magnitude expressed in negative powers of 10 of the difference $\left|\Psi_{x, y}^{20}(u)-\Psi_{x, y}^{18}(u)\right|$; in other words, the number of precision digits, expressed in absolute terms, obtained with approximation $\Psi_{x, y}^{18}(u)$. Calculations were performed using Maple V, release 4 showing a precision of 8-9 digits.

Later, for Pareto claim sizes, the number of significant digits involved in the calculations were raised up to 50 and subsequently the approximations contemplated were finer, $N=40$. As shown in Table 3, approximations then showed $15-19$ precision digits.

Table 2

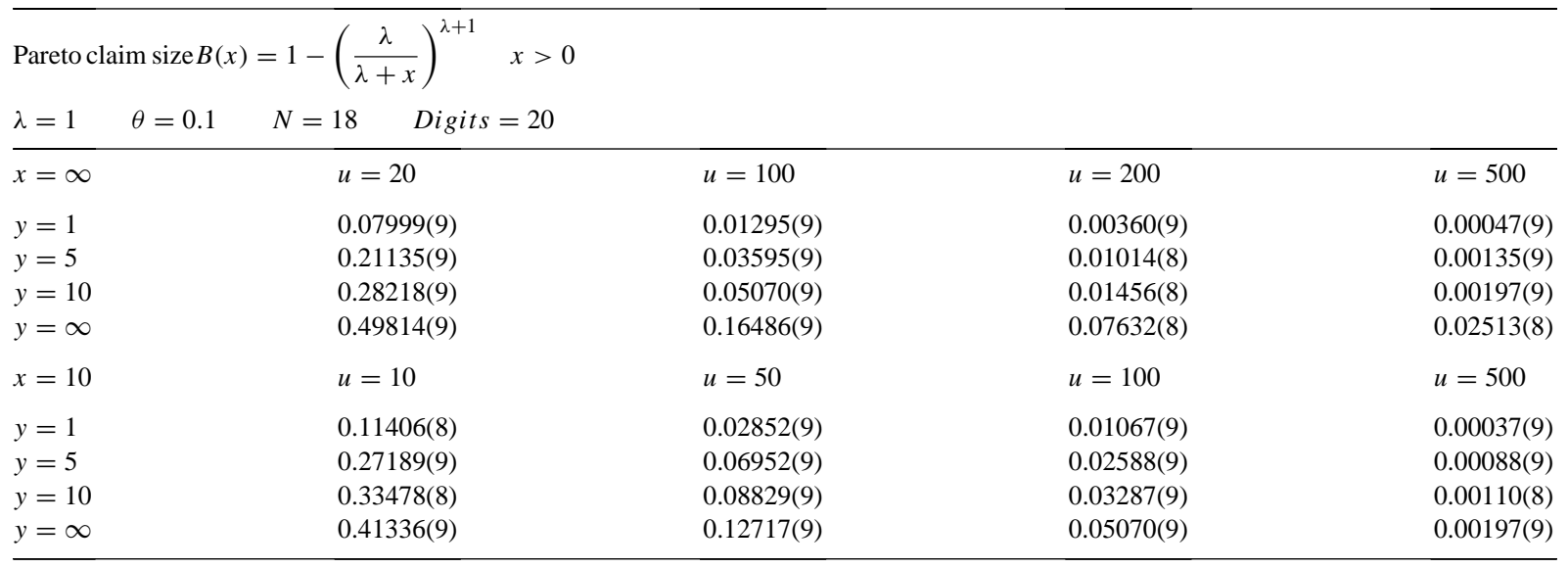




\begin{tabular}{|c|c|c|c|c|}
\hline \multicolumn{5}{|c|}{ Pareto claim size $B(x)=1-\left(\frac{\lambda}{\lambda+x}\right)^{\lambda+1}, \quad x>0$} \\
\hline \multicolumn{5}{|c|}{$\lambda=1 \quad \theta=0.1 \quad N=40 \quad$ Digits $=50$} \\
\hline Magnitude order of & $\left|\Psi_{x, y}^{40}(u)-\Psi_{x, y}^{38}(u)\right|$ & in negative powers of 10 & & \\
\hline$x=\infty$ & $u=20$ & $u=100$ & $u=200$ & $u=500$ \\
\hline$y=1$ & 16 & 17 & 16 & 16 \\
\hline$y=5$ & 16 & 16 & 16 & 16 \\
\hline$y=10$ & 17 & 15 & 15 & 16 \\
\hline$y=\infty$ & 17 & 19 & 17 & 15 \\
\hline$x=10$ & $u=10$ & $u=50$ & $u=100$ & $u=500$ \\
\hline$y=1$ & 15 & 16 & 16 & 16 \\
\hline$y=5$ & 16 & 16 & 15 & 16 \\
\hline$y=10$ & 16 & 15 & 15 & 16 \\
\hline$y=\infty$ & 16 & 16 & 15 & 16 \\
\hline
\end{tabular}

Table 4

\begin{tabular}{|c|c|c|c|c|}
\hline \multicolumn{5}{|c|}{ Log-normal claims size $B(x)=1-\Phi\left(\frac{\ln (x)-\mu}{\sigma}\right)$} \\
\hline$\mu=-1.62$ & $\sigma=1.8$ & $\theta=0.1 \quad N=18$ & Digits $=20$ & \\
\hline$x=\infty$ & & $u=20$ & $u=100$ & $u=200$ \\
\hline$y=1$ & & $0.06067(9)$ & $0.02008(8)$ & $0.00891(9)$ \\
\hline$y=5$ & & $0.19888(9)$ & $0.06808(9)$ & $0.03037(9)$ \\
\hline$y=10$ & & $0.29586(8)$ & $0.10512(9)$ & $0.04723(9)$ \\
\hline$y=\infty$ & & $0.65669(9)$ & $0.34395(9)$ & $0.18812(9)$ \\
\hline$x=30$ & & $u=2$ & $u=10$ & $u=20$ \\
\hline$y=1$ & & $0.15640(9)$ & $0.07988(9)$ & $0.05604(9)$ \\
\hline$y=5$ & & $0.41392(9)$ & $0.24630(9)$ & $0.17751(9)$ \\
\hline$y=10$ & & $0.53423(9)$ & $0.34741(9)$ & $0.25694(8)$ \\
\hline$y=\infty$ & & $0.72652(9)$ & $0.56780(9)$ & $0.46429(9)$ \\
\hline
\end{tabular}

Results can be compared with those in Dickson and Waters (1992), Ramsay (1992a,b) and Ramsay and Usábel (1997) where appropriate.

\section{Concluding comments}

The two most important features of the method presented are that the weights of formula (3.2), $k_{n}^{N}, n=$ $1,2, \ldots, N$ are independent of the claim size distribution or initial reserves considered and that the Laplace transform of the multivariate ultimate ruin probability ought to be evaluated only in the real axis.

The comparative advantage of GS algorithm with respect recursive methods, or other Laplace inversion techniques, is the outstanding reduction of computation time, specially when dealing with heavy-tailed claim size distibutions and large initial reserves. As it is shown in the numerical illustrations, only 18 evaluations of the Laplace transform of the multivariate ruin probability function are necessary to attain $8-9$ absolute precision digits performing calculations with 20 significant digits (see Tables 2 and 4). 
The weights just mentioned above are also the cause of the main weakness of this approach: the magnitude of $k_{n}^{N}$ increases approximately linearly in powers of 10 with increasing values of $N$, as shown in Table 1, becoming unstable when greater values of $N$ are taken into account and the arithmetical precision is not increased at the same time.

However, the use of 30 or more significant digits in classical programs such as FORTRAN, using either Real*16 type or arbitrary precision subroutines, is a common feature in programming (see Section 5). Also computer mathematical assistants based on symbolic algebra and extensively used in actuarial teaching (Maple V or Mathematica or MathLab) allow almost arbitrary precision. In this context, a broader range of values of parameter $N$ can be used, obtaining very fast approximations with a degree of accuracy only attained with extremely huge computation times by recursive methods (see Table 3). Using Gaver-Stehfest Laplace transform inversion technique, the fact of performing calculations with more significant digits is clearly rewarded generously with a very fast rate of convergence $\mathrm{O}((N / 2) !)^{-1}$.

We can finally conclude that this technique used in approximating multivariate ultimate ruin probabilities, for the reasons stated above, can be considered very efficient in terms of computation times, simple, robust and of fast convergence.

\section{Acknowledgements}

I am very grateful to Tim Kampmann of the Deutsche AktuarVereinigung, Professor R. Dalpatadu of the University of Nevada and the Instituto de Actuarios Españoles for their help in bibliography search. I am also indebted to José Marín, Research Fellow of the Department of Mathematics, Heriot-Watt University, Edinburgh for his expert advice regarding FORTRAN and $C^{++}$programming. Financial support from Becas Postdoctorales en el Extranjero, MEC, Spain is gratefully acknowledged.

\section{References}

Bailey, D.H., 1993. Multiprecision translation and execution of FORTRAN programs. ACM Transactions on Mathematical Software 19, $288-319$. Bailey, D.H., 1995. A FORTRAN-90 based multiprecision system. ACM Transactions on Mathematical Software 21, 379-387.

Bohman, H., 1971. Ruin probabilities. Skandinavisk Aktuarietidskrift, pp. 159-163.

Bohman, H., 1974. Fourier inversion-distribution functions - long tails. Scandinavian Actuarial Journal, pp. 43-45.

Bohman, H., 1975. Numerical inversion of characteristic functions. Scandinavian Actuarial Journal, pp. 121-124

Covens, F., Van Wouwe, M., Goovaerts, M., 1979. On the numerical evaluations of Stop-Loss premiums. ASTIN Bulletin 10 (3), 318-324.

Cramér, H., 1955. Collective Risk Theory. F. Skandia (Jubilee Vol.).

Dalpatadu, R.J., Singh, A.K., Tsang, A., 1996. A numerical method for computing the probabiliy distribution of total risk of a portfolio. Actuarial Research Clearing House 1, 481-486.

Davies, B., Martin, B., 1979. Numerical inversion of the Laplace transform: a survey and comparison of methods. Journal of Computational Physics 33, 1-32.

Dickson, D.C.M., 1989. Recursive calculation of the probability and severity of ruin. Insurance: Mathematics and Economics 8, $145-148$.

Dickson, D.C.M., Waters, H., 1992. The probability and severity of ruin in finite and infinite time. ASTIN Bulletin 22, 177-190.

Dickson, C., 1993. On the distribution of the claim causing ruin. Insurance: Mathematics and Economics 12, 143-154.

Dickson, D.C.M., Egidio dos Reis, A.D., Waters, H.R., 1995. Some stable algorithms in ruin theory and their application. ASTIN Bulletin 25, $153-175$.

Dufresne, F., Gerber, H., 1988. The surpluses immediately before and at ruin, and the amount of the claim causing ruin. Insurance: Mathematics and Economics 7, 193-199.

Frey, A., Schmidt, V., 1996. Taylor series expansion for multivariate characteristics of classical risk processes. Insurance: Mathematics and Economics 18, 1-12.

Gaver, D.P., 1966. Operational Research 14, 444-459.

Gelfand, I.M., Shilov, G.E., 1964. Generalized Functions. Academic Press, New York.

Gerber, H., Goovaerts, M., Kaas, R., 1987. On the probability and severity of ruin. ASTIN Bulletin 17, 151-163.

Gradshteyn, I.S., Ryzhik, I.M., 1994. Table of Integrals, Series and Products, 5th ed. Academic Press, New York

Goovaerts, M., De Vylder, F., 1984. A stable recursive algorithm for evaluation of ultimate ruin probabilities. ASTIN Bulletin 14, 53-59.

Panjer, H.H., 1986. Direct calculation of ruin probabilities. Journal of Risk and Insurance 53, 521-529. 
Panjer, H.H., Wang, S., 1993. On the stability of recursive formulas. ASTIN Bulletin 23, 227-258.

Piessens, R., 1969. New quadrature formulas for the numerical inversion of Laplace transforms. BIT 9, 351-361.

Piessens, R., Branders, M., 1971. Numerical inversion of the Laplace transform using generalised Laguerre polynomials. Procceedings of the IEE 118,10

Ramsay, C.M., 1992a. A practical algorithm for approximating the probability of ruin. Transactions of the Society of Actuaries XLIV, $443-459$. Ramsay, C.M., 1992b. Improving Goovaerts and De Vylder's stable recursive algorithm. ASTIN Bulletin 22, 51-59.

Ramsay, C.M., Usábel, M.A., 1997. Calculating ruin probabilities via product integration. ASTIN Bulletin 27, 263-272.

Seal, H., 1971. Numerical calculation of the Bohman-Esscher family of convolution-mixed negative binomial distribution functions. Mitt. Verein. schweiz. Versich.-Mathr. 71, 71-94.

Seal, H., 1974. The numerical calculation of $U(w, t)$, the probability of non-ruin in an interval $(0, t)$. Scandinavian Actuarial Journal, pp. $121-139$. Seal, H.L., 1975. A note on the use of Laguerre polynomials in the inversion of Laplace transforms. Blätter der DAVM 12, 131-134.

Seal, H., 1977. Numerical inversion of characteristic functions. Scandinavian Actuarial Journal, pp. 48-53.

Stehfest, H., 1970. Numerical inversion of Laplace transform. Communications of the ACM. 19, 1.

Thorin, O., 1970. Some remarks on the ruin problem in case the epochs of claims form a renewal process. Skandinavisk Aktuarietidskrift, pp. $29-50$.

Thorin, O., 1971. Further remarks on the ruin problem in case the epochs of claims form a renewal process. Skandinavisk Aktuarietidskrift, $14-38,121-142$.

Thorin, O., 1973. The ruin problem in case the tail of a distribution is completely monotone. Skandinavisk Aktuarietidskrift, pp. 100-119.

Thorin, O., 1977. Ruin probabilities prepared for numerical calculation. Scandinavian Actuarial Journal Supplement, 7-17.

Thorin, O., Wikstad, N., 1973. Numerical evaluation of ruin probabilities for a finite period. ASTIN Bulletin VII (2), 138-153.

Weeks, W.T., 1966. Numerical inversion of Laplace transforms using Laguerre functions. Journal of the ACM 13, 419-426.

Wikstad, N., 1971. Exemplifications of ruin probabilities. ASTIN Bulletin 6, 147-152.

Wikstad, N., 1977. How to calculate ruin probabilities according to the classical risk theory. Scandinavian Actuarial Journal Supplement, 19-24.

Zakian, V., 1969. Electronics Letters 5, 120-121. 